\title{
NUEVA EVIDENCIA DE UN ESTADIO JUVENIL DE PLIOMETANASTES PROTISTUS Y SU RELACIÓN CON ?PLIOMETANASTES GALUSHAI HIRSCHFELD \& WEBB, 1968 (XENARTHRA, MEGALONYCHIDAE) PARA EL MIOCENO SUPERIOR DE COSTA RICA
}

\section{A NEW EVIDENCE OF A JUVENILE ESTATE OF PLIOMETANASTES PROTISTUS AND ITS RELATIONSHIP WITH ?PLIOMETANASTES GALUSHAI HIRSCHFELD \& WEBB, 1968 (XENARTHRA, MEGALONYCHIDAE) FOR THE UPPER MIOCENE OF COSTA RICA}

\author{
Ana L. Valerio ${ }^{1 *} \&$ César A. Laurito ${ }^{2,3}$ \\ ${ }^{1}$ Departamento de Historia Natural, Museo Nacional de Costa Rica. \\ Apdo. 749-1000, San José \\ ${ }^{2}$ INA, Instituto Nacional de Aprendizaje \\ ${ }^{3}$ Investigador Asociado-Departamento de Historia Natural, \\ Museo Nacional de Costa Rica \\ *Autora para contacto: avalerio@museocostarica.go.cr
}

\begin{abstract}
The aim of this paper is to describe a fragment of left mandible of a juvenile specimen of Pliometanastes. That found brings some light about the phyletic relations among Pliometanastes protistus Hirschfeld \& Webb, 1968 and ?Pliometanastes galushai Hirschfeld \& Webb, 1968, and strongly suggests that Pliometanastes is a monospecific genus. Keywords: Mammalia, Xenarthra, Folivora, Pilosa, Pliometanastes, Upper Miocene, Costa Rica.

RESUMEN: La finalidad del presente trabajo es describir un fragmento izquierdo de mandíbula de un espécimen juvenil de Pliometanastes. Dicho hallazgo arroja luz acerca de las relaciones filéticas entre Pliometanastes protistus Hirschfeld \& Webb, 1968 y ?Pliometanastes galushai Hirschfeld \& Webb, 1968 y sugiere de manera notable que el género Pliometanastes es monoespecífico.

Palabras clave: Mammalia, Xenarthra, Folivora, Pilosa, Pliometanastes, Mioceno Superior, Costa Rica.
\end{abstract}

VALERIO, A. L. \& LAURITO, C. A., 2014: Nueva evidencia de un estadio juvenil de Pliometanastes protistus y su relación con ?Pliomenastes galushai Hirschfeld \& Webb, 1968 (Xernarthra, Megalonychidae) para el Mioceno Superior de Costa Rica.- Rev. Geol. Amér. Central, 51: 159-163, DOI: 10.15517/rgac.v51i1.16851 


\section{INTRODUCCIÓN}

En años recientes, se dio a conocer el registro del género Pliometanastes y de su posible especie P. protistus Hirschfeld \&Webb 1968 (sensu Laurito \&Valerio, 2012a) en el Mioceno Superior de Costa Rica. Su hallazgo arrojó luz sobre el arribo temprano de los Megalonychidae a América Central Meridional, mucho antes del cierre del Istmo de Panamá y confirmó a esta región como la ruta de dispersión de éste género y otros xenartros hacia Norte América (Laurito \& Valerio, 2012b). Lo que probablemente ocurrió al final de la época de Colisión temprana o de Syncolisión (Mioceno Medio temprano hasta el Mioceno Tardío, $<14,8 \mathrm{Ma}$ a 7,1 Ma) del Arco de Islas Costa Rica - Panamá con el NW de América del Sur, según los modelos de los escenarios tectónicos planteados por Coates et al. (2004).

Asociado a los restos de Pliometanastes arriba mencionados, se recuperó un pequeño fragmento de una mandíbula de lo que en principio supusimos se trataba de un género diferente de Megalonychidae, que luego de analizarlo exhaustivamente, concluimos que se trataba de un estadio ontogenético juvenil de Pliometanastes que es el objeto de estudio del presente trabajo.

El material procede del sur de Costa Rica, propiamente de la localidad de San Gerardo de Limoncito, Finca Los Laurent, en las coordenadas geográficas 851'19.6”N/8304'51.9”W (Fig. 1).

\section{GEOLOGÍA}

El material recuperado procede de la Formación Curré, propiamente del nivel de conglomerado fino que se ubica hacia el techo de la secuencia de las arcillas azules, que a su vez es sobreyacido por una secuencia de areniscas medias a finas. Lo que confirma su asociación con la subunidad superior o subunidad de lutitas (sensu Mora, 1979).

La litofacies de conglomerados, sedimentológicamente, corresponde con un "ambiente de abanico deltáico subacuático" o "fan delta" y está compuesta por intraclastos de arcilla azul, pro- cedentes del nivel infrayacente al que sobreyace mediante una discordancia erosiva. Los clastos tienen diámetros que varían entre los 5 y $13 \mathrm{~cm}$; estos son bien redondeados, poco o nada esféri$\cos$, algunos presentan contacto puntual, pero el $90 \%$ de ellos flotan en una matriz de arcilla glauconítica. Los clastos se disponen con su eje largo paralelo al plano de estratificación y es posible observar algún grado de imbricación. En esta secuencia son frecuentes los restos óseos y dentales de diversos vertebrados tanto terrestres como marinos (Valerio, 2010). Es de este nivel, cuyo espesor máximo alcanza los $150 \mathrm{~cm}$ y el mínimo observable de $35 \mathrm{~cm}$, donde se recolectó los restos de Pliometanastes.

La edad mínima de la localidad de San Gerardo de Limoncito, fue establecida por Valerio (2010) y Laurito \& Valerio (2010) como Hemphilliano temprano tardío por la asociación de los caballos Protohippus gidleyi Hulbert, 1988, Calippus hondurensis (Olson \& McGrew, 1941) y Dinohippus mexicanus (Lance, 1950).

\section{PALEONTOLOGÍA SISTEMÁTICA}

MAMMALIA Linnaeus, 1758

XENARTHRA Cope, 1889

FOLIVORA Delsuc et al., 2001

PILOSA Flower, 1883

MEGALONYCHIDAE Zittel 1892

Pliometanastes Hirschfeld \& Webb 1968

Pliometanastes protistus Hirschfeld \& Webb 1968

Especie tipo: Pliometanastes protistus Hirschfeld \& Webb 1968

Localidad tipo: McGehee Farm, Alachua County, Florida

Sinonimia:

- Pliometanastes protistus nov. sp. in Hirschfeld \& Webb, 1968, p. 246, figs. 10 a 19. 

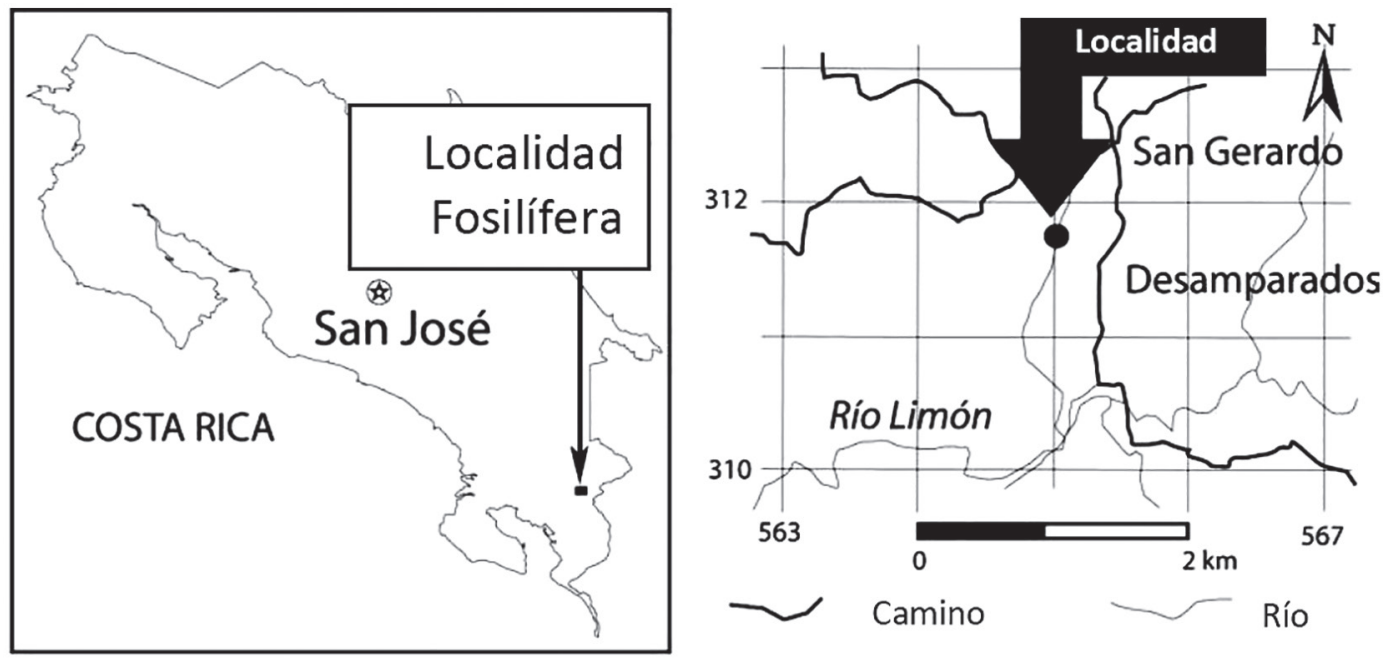

Fig. 1: Mapa de ubicación de la localidad fosilífera de San Gerardo de Limoncito, cantón de Coto Brus, provincia de Puntarenas.

- ?Pliometanastes galushai nov. sp. in Hirschfeld \& Webb, 1968, p. 283, fig. 20.

- Pliometanastes protistus in Hirschfeld, 1981, p. 3, figs. 1-7.

- Pliometanastes cf. P. protistus in Laurito \& Valerio, 2012a, p. 97, figs. 2 y 3.

Material: CFM-2916, fragmento mandibular izquierdo, sin ramo ascendente con preservación parcial del alveolo del $1 \mathrm{mf}$ y preservación del $2 \mathrm{mf}$ y $3 \mathrm{mf}$ (Fig. 2a-d). El ejemplar se encuentra depositado en la Colección de Fósiles de la Sección de Geología del Museo Nacional de Costa Rica.

Descripción: la sección de la rama horizontal de la mandíbula correspondiente a los molarifores $1 \mathrm{mf}$ a $3 \mathrm{mf}$, es corta, alta y gruesa, cara externa o labial ligeramente inflada y amplio canal mandibular; molariformes insertos verticalmente; apertura posterolateral del canal mandibular ubicado en el extremo superior a nivel del primer tercio anterior del $3 \mathrm{mf}$ $\mathrm{y}$ en la base del ramo ascendente. El fragmento de mandíbula tiene una longitud de 47,07 $\mathrm{mm}$ y un ancho de 22,50 mm. El $2 \mathrm{mf}$ es subrectangular con los bordes redondeados y está dispuesto de manera perpendicular con respecto al eje largo del ramo mandibular; tiene 13,34 mm de longitud vestíbulo-lingual y $10,33 \mathrm{~mm}$ de longitud antero-posterior, la cara labial de este molariforme es ligeramente convexa y más ancha que la cara lingual que es más angosta y redondeada ello le imprime un contorno trapezoidal al diente. El $3 \mathrm{mf}$ es subrectangular ligeramente cuadrangular y está dispuesto de manera oblicua con respecto al eje largo del ramo mandibular, tiene $12,41 \mathrm{~mm}$ de longitud-vestíbulo lingual y $10,85 \mathrm{~mm}$ de longitud antero-posterior.

\section{DISCUSIÓN Y CONCLUSIONES}

En el año de 1947, el paleontólogo estadounidense Ted Galusha, recolectó un fragmento de mandíbula de un Megalonychidae juvenil en la localidad de San Juan al norte de la ciudad de Española, en el Estado de Nuevo México, el mismo fue estudiado por Hirschfeld \& Webb (1968), quienes crean la especie ?Pliometanastes galushai y dejan abierta la posibilidad de que se trate de un ejemplar subadulto de Pliometanastes protistus. Desde entonces, no se han encontrado, ni descrito nuevos restos de ?Pliometanastes galushai, sean estos hallazgos aislados o asociados a ejemplares adultos de la misma especie o de Pliometanastes protistus.

Curiosamente el ejemplar de Pliometanastes recuperado en la localidad de San Gerardo de Limoncito, presenta caracteres de un ejemplar juvenil como lo son el pequeño tamaño de la 

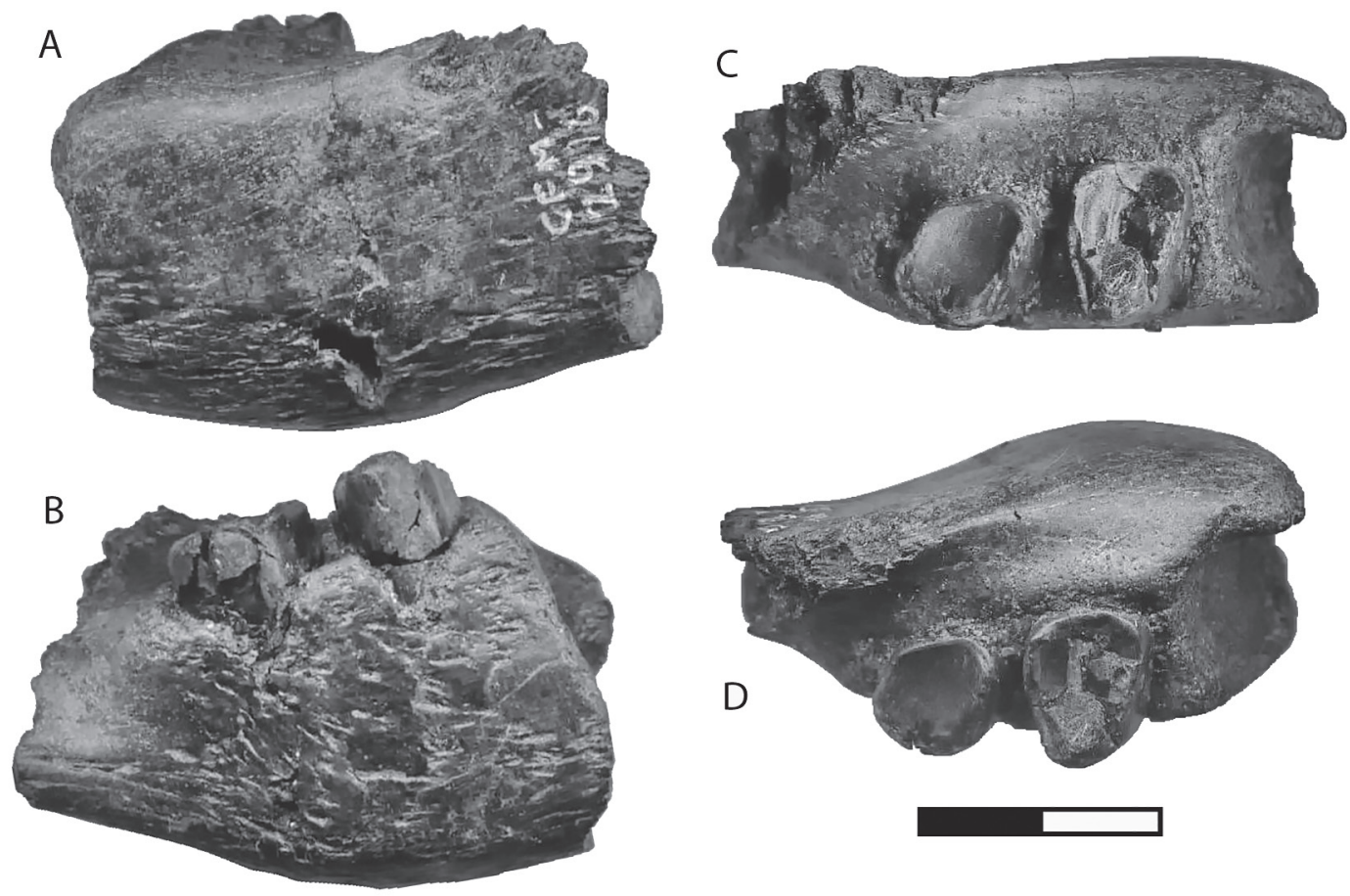

Fig. 2: Pliometanastes protistus, fragmento de mandíbula en vistas: a. labial, b. lingual, c. oclusal y d. oclusal ligeramente oblicua. Escala visual $2 \mathrm{~cm}$.

mandíbula, cuyo hueso es particularmente poroso y molares con superficie oclusal muy plana, aparte de que la cavidad del $1 \mathrm{mf}$ sugiere que este molar era más ancho hacia la base, en vez de mantener el mismo diámetro a lo largo de toda su longitud, características típica de los Megalonychidae subadultos.

Por otra parte, el contorno del $2 \mathrm{mf}$ del ejemplar costarricense es muy similar al $\mathrm{mf} 2$ descrito para ?Pliometanastes galushai, lo que sugiere fuertemente de que se trata de la misma especie. En cuanto a las dimensiones, aunque los 2 son más anchos que largos, el ejemplar del presente estudio es notablemente mayor que el ejemplar de Nuevo México (13,34 mm x 10,33 mm vs 10,00 $\mathrm{mm} \times 5,3 \mathrm{~mm}$ ). Esta diferencia de dimensiones permite asumir que se trata de 2 estadios ontogenéticos diferentes.

Ahora bien, se puede concluir de manera certera que el ejemplar CFM-2916 es un subadulto de Pliometanastes protistus porque se encontró asociado a varios restos óseos y dentales de adultos de dicha especie y porque el yacimiento fosilífero de San Gerardo de Limoncito se caracteriza por una marcada tafonomía atricional, es decir por el registro selectivo de individuos que murieron muy jóvenes o viejos.

Se puede también concluir, pero de manera subjetiva, que al no volverse a registrar ejemplares que confirmen a la especie ?Pliometanastes galushai Hirschfeld \& Webb, 1968, sean especímenes juveniles aislados o asociados a algún adulto, que dicha especie en realidad está fundamentada en un ejemplar subadulto de otra especie de Pliometanastes y en este caso de Pliometanastes protistus Hirschfeld \& Webb, 1968.

Por último, el ejemplar CFM-2916 que presenta gran similitud con ?Pliometanastes galushai Hirschfeld \& Webb, 1968, y este hecho solo permite concluir que ambas especies son sinónimas y que el género Pliometanastes en realidad es monoespecífico. 


\section{REFERENCIAS BIBLIOGRÁFICAS}

COATES, A. G., COLLINS, L. S., AUBRY, M. \& BERGGREN, W. A., 2004: The Geology of the Darien, Panama, and the late MiocenePliocene collision of the Panama arc with northwestern South America.- Geol. Soc. Amer. Bull. 116: 1327-1344.

DELSUC, F., CATZEFLIS, F. M, STANHOPE, M. J. \& DOUZERY, E. J. P., 2001: The evolution of armadillos, anteaters and sloths depicted by nuclear and mitochondrial phylogenies: Implications for the status of the enigmatic fossil Eurotamandua.Proc. Biol. Sci. 268(1476): 1605-1615.

HIRSCHFELD, S.E., 1981: Pliometanastes protistus (Edentata, Megalonychidae) from Knight's Ferry, California, with discussions of Early Hemphillian Megalonychids. PaleoBios, 36: 1-16.

HIRSCHFELD, S. E. \& WEBB, S. D., 1968: PIioPleistocene megalonychid sloths of North America.- Bull. of the Fla. State Mus. Biol. Sci. 12(5): 213-296.

HULBERT, R. C., 1988: Calippus and Protohippus (Mammalia, Perissodactyla, Equidae) from the Miocene (BarstovianEarly Hemphillian) of the Gulf Coastal Plain.- Bull. Florida State Mus. Biol. Sci. 32(3): 221-340.

LANCE, J. F., 1950: Paleontología y Estratigrafía del Plioceno de Yepómera, Estado de Chihuahua $1^{\text {a }}$ parte: Equidos, excepto Neohipparion.- Univ. Nac. Autónoma de México, Inst. de Geol. 54: 1-81.
LAURITO, C. \& VALERIO, A., 2010: Los caballos fósiles de la Formación Curré, cantón de Coto Brus, Costa Rica.- 131 págs. Museo Nacional de Costa Rica, San José.

LAURITO, C. A., \& VALERIO, A. L. 2012a: Primer registro fósil de Pliometanastes sp. (Mammalia, Xenarthra, Megalonychidae) para el Mioceno Superior de Costa Rica, América Central. Una Nueva Pista en la Comprensión del Pre-GABI.- Rev. Geol. Amér. Central, 47: 95-108.

LAURITO, C. \& VALERIO, A., 2012b: Paleobiogeografía del arribo de mamíferos suramericanos al sur de América Central previo al gran intercambio biótico americano: Un vistazo al GABI en América Central.- Rev. Geol. Amér. Central, 46: 123-144.

MORA, S., 1979: Estudio geológico de una parte de la región sureste del Valle del General, provincia de Puntarenas, Costa Rica.- 157 págs. Univ. de Costa Rica, San José [Tesis Lic.].

OLSON, E. C. \& MCGREW, P. O., 1941: Mammalian fauna from the Pliocene of Honduras.- Bull. Geol. Soc. America, 52: 1219-1244.

VALERIO, A. L., 2010: Paleontología, bioestratigrafía y paleoecología de los caballos fósiles de la Formación Curré en el Cantón de Coto Brus, Costa Rica (análisis basado en material dental).- 353 págs. Univ. de Costa Rica, San José [Tesis Lic.]. 